\title{
Imaging early endothelial inflammation following stroke by core shell silica superparamagnetic glyconanoparticles that target selectin
}

Tracy D. Farr, ${ }^{1, *}$ Chian-Hui Lai, ${ }^{2,}{ }^{\ddagger}$ Dan Grünstein, ${ }^{2}$ Guillermo Orts-Gil, ${ }^{2}$ Cheng-Chung

Wang, ${ }^{2, \dagger}$ Philipp Boehm-Sturm, ${ }^{1}$ Peter H. Seeberger, ${ }^{2,3} *$ Christoph Harms ${ }^{1, *}$

${ }^{1}$ Department of Experimental Neurology, Center for Stroke Research Berlin (CSB), Charité

University Medicine, Charitéplatz 1, 10117 Berlin, Germany.

${ }^{2}$ Department of Biomolecular Systems, Max-Planck-Institute of Colloids and Interfaces, Am

Mühlenberg 1, 14476 Potsdam-Golm, Germany.

${ }^{3}$ Freie Universität Berlin, Institute of Chemistry and Biochemistry, Arnimallee 22, 14195

Berlin, Germany.

KEYWORDS: Glyco-magnetic nanoparticle, MRI, stroke, selection 


\section{ABSTRACT}

Activation of the endothelium is a pivotal first step for leukocyte migration into the diseased brain. Consequently, imaging this activation process is highly desirable. We synthesized carbohydrate-functionalized magnetic nanoparticles that bind specifically to the endothelial transmembrane inflammatory proteins $\mathrm{E}$ and $\mathrm{P}$ selectin. Magnetic resonance imaging revealed that the targeted nanoparticles accumulated in the brain vasculature following acute administration into a clinically relevant animal model of stroke, though increases in selectin expression were observed in both brain hemispheres. Non-functionalized naked particles also appear to be a plausible agent to target the ischemic vasculature. The importance of these findings is discussed regarding the potential for translation into the clinic.

Inflammation plays a major role in neurological disorders such as stroke, and the ability to image inflammatory processes would provide valuable information to aid patient stratification, and eventually treatment. Magnetic resonance imaging (MRI) is being used increasingly as a diagnostic tool in the acute stroke setting, and several contrast agents are approved to analyze the injured brain for blood brain barrier breakdown or vascularization. Magnetic nanoparticles (MNPs), especially iron oxide $\mathrm{Fe}_{3} \mathrm{O}_{4}-\mathrm{MNPs}$, have been widely used as biosensors owing to their relatively low toxicity. ${ }^{1-5}$ Some MNP formulations are potential 
imaging agents for the inflammatory response to stroke, particularly the blood borne leukocytes. ${ }^{6}$ However, lack of blood brain barrier penetrability and relatively poor inflammatory cell uptake has remained an obstacle. ${ }^{7,8}$ Therefore, the identification and specific targeting of accessible inflammatory components, for example, the endothelium, is highly attractive. E and $\mathrm{P}$ selectin (CD62E and P, respectively) are endothelial transmembrane proteins that participate in leukocyte adherence to blood vessels during inflammation. ${ }^{9} \mathrm{E}$ and $\mathrm{P}$ selectin are generally thought to be pro-inflammatory, and thus detrimental to stroke outcome, hence, they have been suggested as potential imaging targets. ${ }^{10}$ For selectin binding, MNPs have been functionalized with peptides $^{5}$ and carbohydrates such as Sialyl Lewis ${ }^{\mathrm{X}}$ (SX). ${ }^{1}$ Carbohydrate-functionalized MNPs can take advantage of clustering effects to achieve high binding affinities and specificities when compared to monovalent carbohydrate-protein interactions. ${ }^{11-13}$ Several glyco-functionalized nanoparticles (NPs) with high surface to volume ratios have been reported to be good multivalent carriers of carbohydrate ligands that bind with high affinity to the targeted receptors. ${ }^{14-19}$ We designed silica coated iron oxide core shell MNPs, which exhibit superparamagnetism and strong saturation magnetism, and also lend themselves to carbohydrate functionalization. ${ }^{4,15}$ The silica outer shell provides excellent stability and protection of the magnetic core, thus avoiding oxidative stress to cells. ${ }^{2,20}$

The known E and P selectin ligand sialyl Lewis ${ }^{\mathrm{x}}(\mathrm{SX}, 1)$ was immobilized on silica iron oxide nanoparticles (SX@MNPs) to target these proteins that are believed to be expressed on 
activated vascular endothelial cell as a result of inflammation. The interactions between the selectins and SX-glycoconjugate ligands play key roles in adhesive interaction among these inflamed vascular surfaces. As non-targeting controls for targeting experiments using SX@MNPs, nanoparticles carrying the carbohydrate Lewis ${ }^{\mathrm{X}}$ (LX, 2) (LX@MNP) or simple hydroxy groups (HO@MNPs) were designed. The three different MNP formulations were administered to mice that underwent transient middle cerebral artery occlusion (MCAO), which is a clinically relevant animal model of stroke with a strong inflammatory component.

Amino-functionalized silica iron oxide core shell nanoparticles $\left(\mathbf{N H}_{\mathbf{2}} @ \mathbf{M N P s}\right)$ with a diameter of 18 nm (Figure 1a) were prepared as described. ${ }^{15} \mathbf{N H}_{2} @ \mathbf{M N P s}$ were then reacted with succinic anhydride under basic conditions at room temperature overnight to afford carboxylic acid modified nanoparticles (COOH@MNPs, Scheme 1). Activation of the carboxylic acid groups on the surface of the COOH@MNPs was accomplished using NHS and EDC to yield $N$-hydroxysuccinimide activated MNPs (NHS@MNPs). After removing excess reagents, $\mathrm{LX}^{21}$ and $\mathrm{SX}^{22}$ were immediately added to the NHS@MNPs in DMF and $\mathrm{NEt}_{3}$ at room temperature for $48 \mathrm{~h}$. LX 1 and SX 2 were prepared using an automated solid-phase oligosaccharide synthesizer ${ }^{21,22}$ (NMR spectra see Figures S1 and S2). Following amide bond formation, the solvent was removed by magnetic separation and the remaining NHS groups on the MNPs were capped with ethanolamine to afford carbohydrate decorated 
MNPs (LX@MNPs and SX@MNPs, respectively). In a similar manner HO@MNPs were prepared by quenching the NHS-groups without the addition of carbohydrates.

Scheme 1. Synthesis of HO@MNPs and carbohydrate@MNPs (LX@MNPs and

\section{SX@MNPs).}

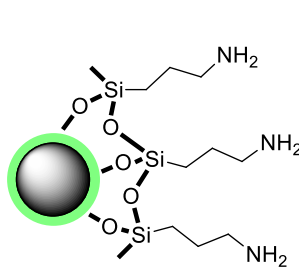

$\mathrm{NH}_{2} @ M N P$
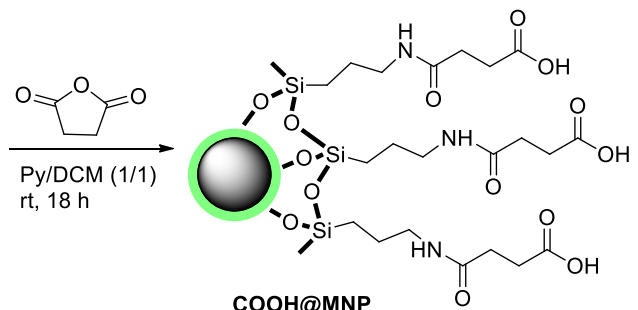

COOH@MNP

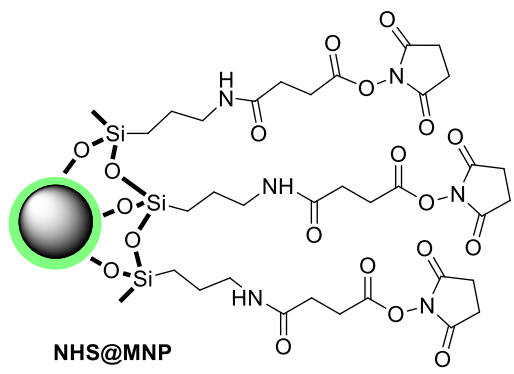
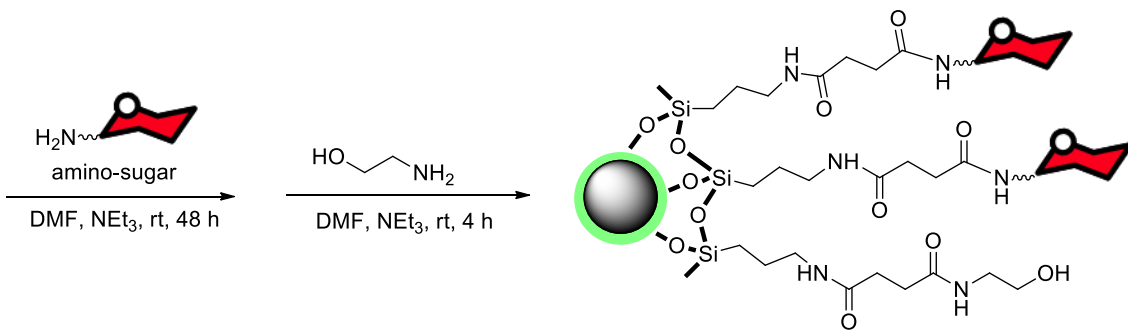

LX@MNP (R= Lewis $X)$

SX@MNP $(R=$ Siayl Lewis $X)$

HO@MNP (Without adding sugar ligand)
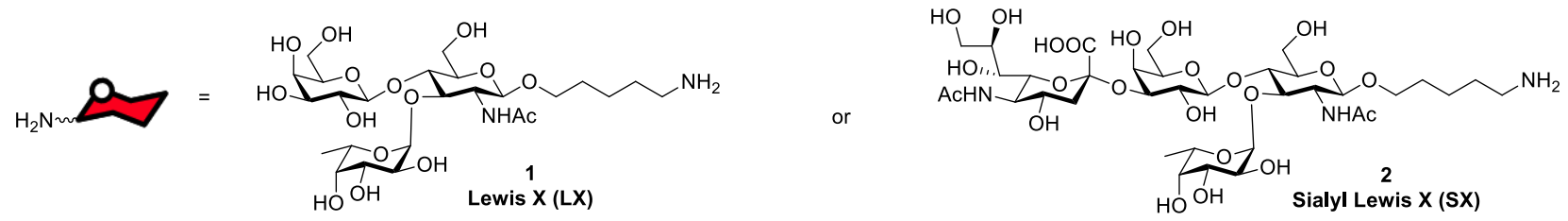

All MNP formulations were characterized by the zeta potential for their surface charge

(Figure 1b) and dynamic light scattering (DLS) for their hydrodynamic diameter (Figure S3 and Table S1). The zeta-potential measurements (Figure 1b) provided valuable information 
about the success of each reaction step. Intensity-weighted hydrodynamic diameters were found to be between 120 and $160 \mathrm{~nm}$ and the polydispersity indexes were between 0.17 and 0.22. The amount of SX present on the SX@MNPs was estimated using a SialicQ quantification kit (Figure S4). This enzymatic method can detect the efficiency of sialic acid incorporation and can be accurately measured using spectrophotometry. Levels of Sialyl LewisX in the SX@MNPs were determined to be at $10.5 \pm 1.2 \mathrm{nmol}^{\circ} \mathrm{mg}^{-1}$, corresponding to around 30\% coupling yield over three reaction steps based on the density of the amines in $\mathbf{N H}_{2} @$ MNPs. ${ }^{15}$ Binding specificity of the SX@MNPs to rat E-Selectin was tested in vitro using a spectrophotometric binding method (Figures 1c and S5) and SX@MNPs resulted in the strongest binding to the E-selectin-Fc chimera protein. 
a)

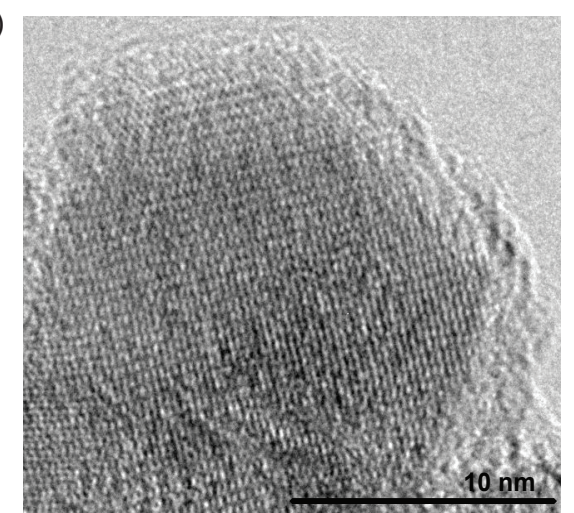

b)

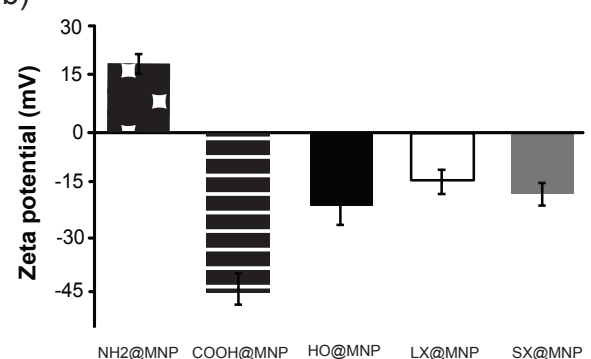

c)

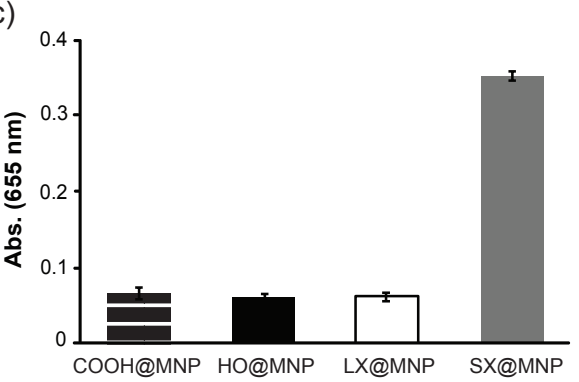

Figure 1. (a) TEM image of $\mathbf{N H}_{2} @$ MNPs. (b) Zeta-potential of the particle formulations indicating overall charge (NUMBER OF SAMPLES). (c) Specific binding assay of each MNP formulation to rat E-selectin in vitro (NUMBER OF SAMPLES).

Following extensive MNP characterization, we randomly administered one of the three formulations (HO@MNPs, LX@MNPs, or SX@MNPs) to mice 5.5 h after they 
underwent the most common animal model of stroke: transient MCAO via the intraluminal filament technique (Supplementary Methods). A transient model of MCAO was chosen because reperfusion occurs frequently in the patient population. Furthermore, transient models reduce the risk of intravascular trapping of contrast agents in situations of low flow that are produced with permanent models. The intraluminal filament technique offers the additional advantage of not challenging the endothelium of the middle cerebral artery (MCA) as would be the case with vasoconstrictors. $\mathrm{T}_{2}{ }^{*}$ weighted sequences were chosen to identify the MNPs as they are extremely sensitive to susceptibility effects. $A \mathrm{~T}_{2} *$ weighted sequence was acquired just prior to MNP administration (5 h post MCAO) to exclude the presence of pre-existing microbleeds and/or endogenous sources of negative contrast. The sequence was repeated immediately after intravenous MNP administration to detect blood pool effects, and again at $24 \mathrm{~h}$ post MCAO to observe vascular accumulation. Massive blood pool effects were observed following administration of all MNP formulations in the $\mathrm{T}_{2}{ }^{*}$ weighted images, which were no longer present at $24 \mathrm{~h}$. However, at this time, numerous punctate regions of signal loss were observed in the brains of all animals (Figure 2). 
a) HO@MNP

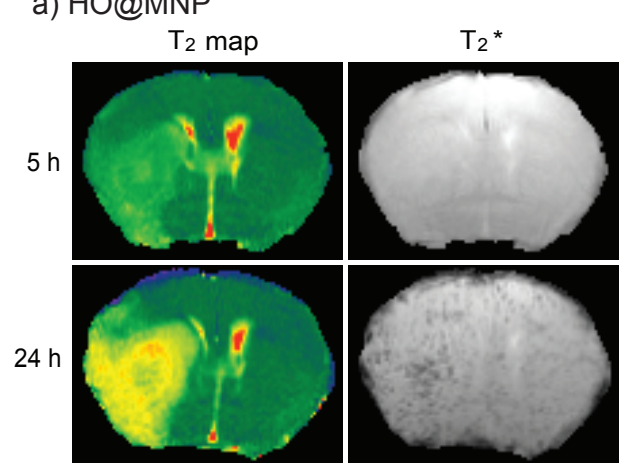

b) LX@MNP

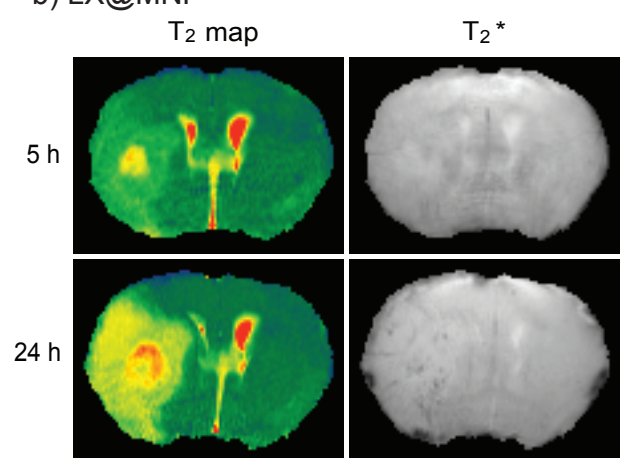

c) SX@MNP

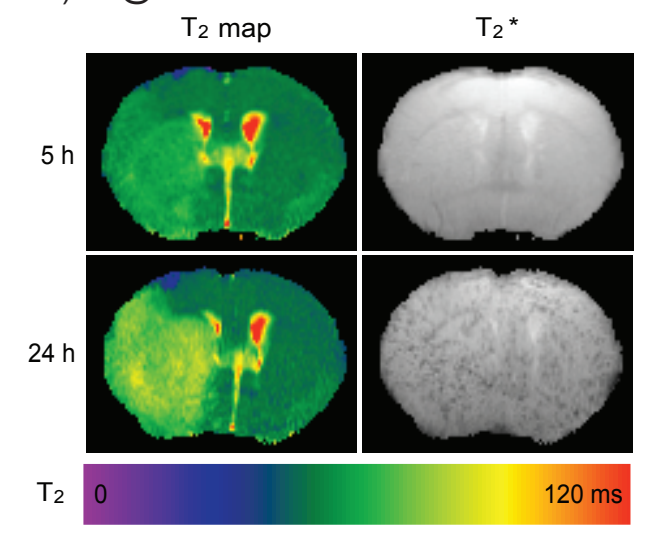

Figure 2. $\mathrm{T}_{2}$ maps and $\mathrm{T}_{2} *$ weighted images at 5 and $24 \mathrm{~h}$ after MCAO in a representative animal that received (a) HO@MNPs (n=5), (b) LX@MNPs (n=6), or (c) SX@MNPs (n=5).

Note: the color scale bar corresponds to the $\mathrm{T}_{2}$ value $(\mathrm{ms})$. 
$T_{2}$ maps and $T_{1}$ weighted images were acquired in parallel to the $T_{2}{ }^{*}$ weighted images. $T_{2}$ maps detect and quantify ischemic damage that is only beginning to develop at $5 \mathrm{~h}$ post MCAO and is maximal at $24 \mathrm{~h}$. There were no differences in infarct sizes among the groups at either time point ( 5 or $24 \mathrm{~h}$ post MCAO), indicating comparable levels of pathology among the animals. The ischemic tissue is not visible with $\mathrm{T}_{1}$ weighting, thus making it easier to observe relaxivity changes due to the MNPs. However, the effects were most pronounced, as anticipated, in the $\mathrm{T}_{2}{ }^{*}$ weighted images.

To confirm which groups exhibited the greatest accumulation of MNPs at $24 \mathrm{~h}$ post $\mathrm{MCAO}$, the number of punctate regions of signal loss were counted in the entire brain, as well as within the ischemic territory and the corresponding region mirrored to the contralateral hemisphere (Figure 3). 
a)

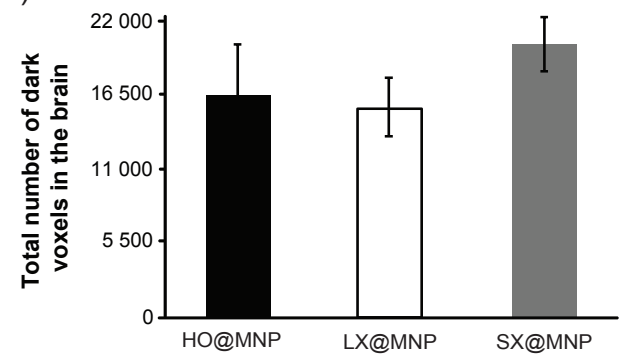

b)

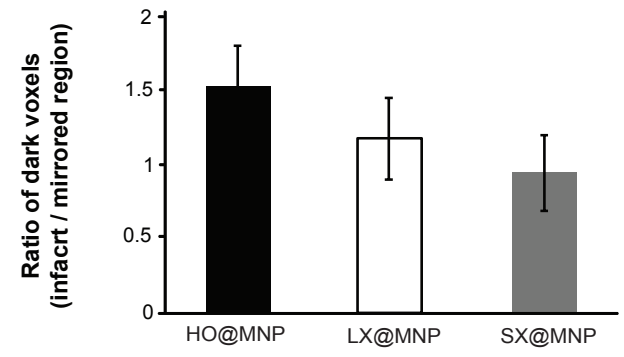

c)

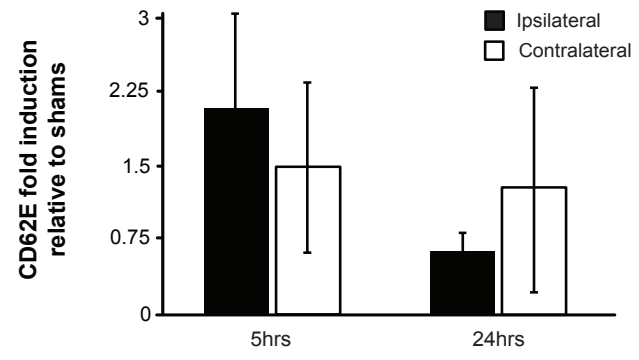

Figure 3. a) The total number of dark voxels in the brains of the animals from each group:

HO@MNPs (n=5), LX@MNPs (n=6), and SX@MNPs (n=5). b) The number of dark voxels in the infarcted tissue expressed as a ratio of the mirrored contralateral region. c) The amount of CD62E expression (in relation to sham animals $(n=3)$ ) in the ipsi- and contralateral hemispheres of mice with either $5(n=4)$ or $24 \mathrm{~h}(\mathrm{n}=4)$ of MCAO.

There was a significant and robust main effect of group $\left(F(2,13) 4.536, p=0.03 ; \omega^{2}=0.1\right.$; one way ANOVA) when the total number of dark voxels across the entire brain was compared 
(Figure 3a). The largest number of dark voxels overall in the brain were observed in the $\mathbf{S X} @ \mathbf{M N P}$ treated group and the lowest number in the LX@MNP group ( $\mathrm{p}=0.036$, post-hoc comparison; $d=2.167$ ) (Figure 3a). However, even though there were more dark spots in the $\mathbf{S X} @ \mathbf{M N P}$ treated group, the difference between this group and the HO@MNP treated group failed to reach significance. These in vivo observations partially support the in vitro binding specificity results in that SX@MNPs are more abundant when compared to the non-functionalized LX@MNP control particles, but not the HO@MNP particles.

There was also a significant and robust main effect of group $\mathrm{F}(2,13) 5.997, \mathrm{p}=0.014$; $\omega^{2}=0.2$; one way ANOVA) when the number of dark voxels (expressed as a ratio between the ischemic tissue and the corresponding mirrored region in the intact side of the brain) was compared (Figure 3b). The highest ratio was observed in the HO@MNP treated group, while the lowest ratio was observed in the SX@MNP group ( $\mathrm{p}=0.013$, post-hoc comparison; $\mathrm{d}=$ 2.215) (Figure 3b). This may be partially explained by the fact that there were two animals in the HO@MNP treated group that exhibited large regions of signal loss at $24 \mathrm{~h}$ in the ischemic territory (Figure S6) that we have not seen with a variety of other non-functionalized MNPs. ${ }^{7}$ HO@MNPs may therefore be prone to accumulation in the ischemic territory irrespective of selectin expression. It is possible that there is an unknown factor in the in vivo microenvironment that is responsible for this, or that the surface polarity of these particles 
differs. Indeed, a comparison of the three particles indicates that HO@MNPs are less polar. On the other hand, the lack of an increased number of dark spots in the ischemic tissue of the SX@MNP treated group suggests that increases in selectin expression may not be confined to the injured tissue, a phenomenon we confirmed independently using a Western blot to semi-quantify CD62E expression in the ischemic and contralateral hemispheres of mice that underwent MCAO. At $5 \mathrm{~h}$ post $\mathrm{MCAO}$, there was only a slight increase in CD62E expression in the ischemic hemisphere, which was not significant (Figure 3c). By $24 \mathrm{~h}$, selectin expression in the contralateral hemisphere was still comparable to that of the contralateral hemisphere at $5 \mathrm{~h}$. However, CD62E expression was lower in the ipsilateral hemisphere, which is likely explained by the extensive damage incurred to the ischemic tissue by $24 \mathrm{~h}$. However, this decrease was still not significantly different from the expression in the contralateral side. These findings are not completely unexpected as increased selectin expression in the intact hemisphere following MCAO has been reported. ${ }^{23}$ Indeed, stroke is also a systemic disease with upregulation of the peripheral inflammatory system and blood borne pro-inflammatory mediators that have widespread effects.

To exclude the possibility that the MNPs were entering the brain parenchyma rather than accumulating in the blood vessels, brain tissue was extracted and stained with Prussian blue to detect the presence of iron (Figure 4). 


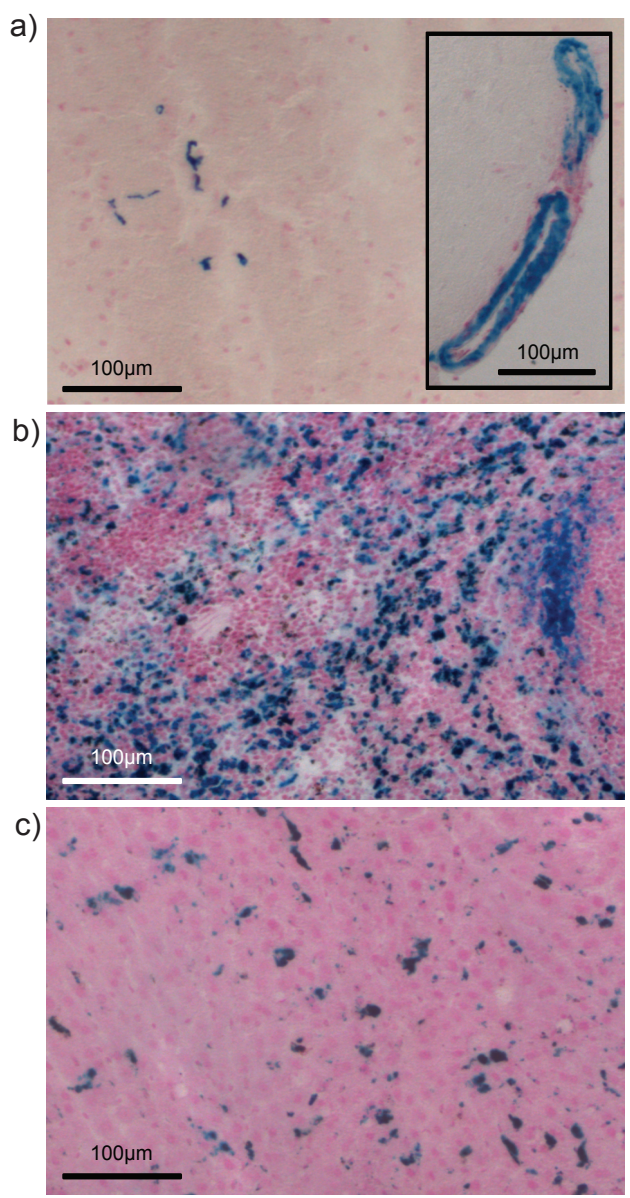

Figure 4. a) Prussian blue staining in blood vessels in the ischemic territory of an animal treated with HO@MNPs. b) High amounts of iron were detected in the spleen and c) liver.

Prussian blue staining confirmed that most of the iron was found in the spleen (Figure 4b) and liver (Figure 4c) of the animals in all three treatment groups. Iron was not detected in the brains of any of the animals, which implies that binding or accumulation in the blood vessels was disrupted during tissue preparation, likely due to the saline perfusion. However, a small amount of iron was identified in the ischemic region of a single animal that received HO@MNPs (Figure 4a). In this animal, we confirmed that the MNPs were confined to the blood vessels and not the parenchyma (Figure 4a inset). 
In conclusion, we investigated the ability of SX@MNPs to target E and P selectin in the ischemic brain. While the specificity of these particles appears to be high for the target, selectin expression is up-regulated over the entire brain, and not only in the ischemic tissue. Interestingly, when appropriate control particles are employed, HO@MNPs appear to be best at accumulating in the injured blood vessels. This highlights both the inherent challenges that arise from in vivo work with increasingly complicated models and the utility of appropriate control experiments. Caution is therefore urged during interpretation on account of the variability. As the employed MRI sequences can be easily applied in the clinic, these results are at the forefront of translation moving towards non-invasive imaging of the activated endothelium in a patient population.

\section{ASSOCIATED CONTENT}

Supporting information available: Detailed materials and methods section and supplementary

figures. This material is available free of charge via the Internet at http://pubs.acs.org.

\section{AUTHOR INFORMATION}

\section{Corresponding Authors}

*E-mail: Peter.Seeberger@mpikg.mpg.de

*E-mail: christoph.harms@charite.de 


\section{Present Addresses}

$\dagger$ Present address: Institute of Chemistry, Academia Sinica, Taipei, 11529, Taiwan.

\section{Author Contributions}

† T. D. F. and C.-H. L. contributed equally.

\section{ACKNOWLEDGMENT}

We thank the Max-Planck Society for generous financial support. C.-H. Lai thanks the National Science Council (Taiwan) and DAAD for a stipend. D. G. thanks the European Union FP7 (CARMUSYS to P. H. S.) for financial support. The authors wish to acknowledge Janet Lips and Nadine Weser for technical assistance and Dr. Davide Esposito for assistance in preparing the carbohydrate building blocks. This work was supported by grants from the Federal Ministry of Education and Research (Center for Stroke Research Berlin) (TDF, CH), and the German Research Foundation (Clinical Research Group KFO213 to $\mathrm{CH}$, HA-5741/1-1 and HA5741/1-2).

\section{REFERENCES}

(1) van Kasteren, S. I.; Campbell, S. J.; Serres, S.; Anthony, D. C.; Sibson, N. R.; Davis, B. G. Proc. Natl. Acad. Sci. U.S. A. 2009, 106, 18-23.

(2) Zhou, L.; Yuan, J.; Wei, Y. J. Mater. Chem. 2011, 21, 2823-2840.

(3) Lee, N.; Choi, Y.; Lee, Y.; Park, M.; Moon, W. K.; Choi, S. H.; Hyeon, T. Nano Lett. 2012, 12, 3127-3131.

(4) El-Boubbou, K.; Zhu, D. C.; Vasileiou, C.; Borhan, B.; Prosperi, D.; Li, W.; Huang, X. J. Am. Chem. Soc. 2010, 132, 4490-4499. 
(5) Jin, A. Y.; Tuor, U. I.; Rushforth, D.; Filfil, R.; Kaur, J.; Ni, F.; Tomanek, B.; Barber, P. A. Contrast Media Mol. Imaging 2009, 4, 305-311.

(6) Beckmann, N.; Cannet, C.; Babin, A. L.; Blé, F.-X.; Zurbruegg, S.; Kneuer, R.; Dousset, V. Wiley Interdiscip. Rev. Nanomed. Nanobiotechnol. 2009, 1, 272-298.

(7) Harms, C.; Datwyler, A. L.; Wiekhorst, F.; Trahms, L.; Lindquist, R.; Schellenberger, E.; Mueller, S.; Schutz, G.; Roohi, F.; Ide, A.; Fuchtemeier, M.; Gertz, K.; Kronenberg, G.; Harms, U.; Endres, M.; Dirnagl, U.; Farr, T. D. J. Cerebr. Blood F. Met. 2013, 33, 795-796.

(8) Farr, T. D.; Seehafer, J. U.; Nelles, M.; Hoehn, M. NMR Biomed. 2011, 24, 35-45.

(9) Suzuki, H.; Abe, K.; Tojo, S.; Kimura, K.; Mizugaki, M.; Itoyama, Y. Neurol. Res. 1998, 20, 463-469.

(10) Yilmaz, G.; Granger, D. N. Neurol. Res. 2008, 30, 783-793.

(11) Lundquist, J. J.; Toone, E. J. Chem. Rev. 2002, 102, 555-578.

(12) Gestwicki, J. E.; Cairo, C. W.; Strong, L. E.; Oetjen, K. A.; Kiessling, L. L. J. Am. Chem. Soc. 2002, 124, 14922-14933.

(13) Mathai Mammen, S.-K. C., George M. Whitesides. Angew. Chem., Int. Ed. 1998, 37, 2754-2794.

(14) Lin, C. C.; Yeh, Y. C.; Yang, C. Y.; Chen, C. L.; Chen, G. F.; Chen, C. C.; Wu, Y. C. J. Am. Chem. Soc. 2002, 124, 3508-3509.

(15) Lai, C.-H.; Lin, C.-Y.; Wu, H.-T.; Chan, H.-S.; Chuang, Y.-J.; Chen, C.-T.; Lin, C.-C. Adv. Funct. Mater. 2010, 20, 3948-3958.

(16) Lai, C.-H.; Chang, T.-C.; Chuang, Y.-J.; Tzou, D.-L.; Lin, C.-C. Bioconjugate Chem. 2013, 24, 1698-1709.

(17) Lai, C.-H.; Lai, N.-C.; Chuang, Y.-J.; Chou, F.-I.; Yang, C.-M.; Lin, C.-C. Nanoscale 2013, 5, 9412-9418.

(18) Kikkeri, R.; Lepenies, B.; Adibekian, A.; Laurino, P.; Seeberger, P. H. J. Am. Chem. Soc. 2009, 131, 2110-2112.

(19) Kennedy, D. C.; Grunstein, D.; Lai, C. H.; Seeberger, P. H. Chem.- Eur. J. 2013, 19, 3794-800.

(20) Wu, J.; Ding, T.; Sun, J. Neurotoxicology 2013, 34, 243-253.

(21) Schlegel, M. K.; Hutter, J.; Eriksson, M.; Lepenies, B.; Seeberger, P. H. Chembiochem 2011, 12, 2791-2800.

(22) Krock, L.; Esposito, D.; Castagner, B.; Wang, C.-C.; Bindschadler, P.; Seeberger, P. H. Chem. Sci. 2012, 3, 1617-1622.

(23) Haring, H.-P.; Berg, E. L.; Tsurushita, N.; Tagaya, M.; del Zoppo, G. J. Stroke 1996, 27, 1386-1392. 


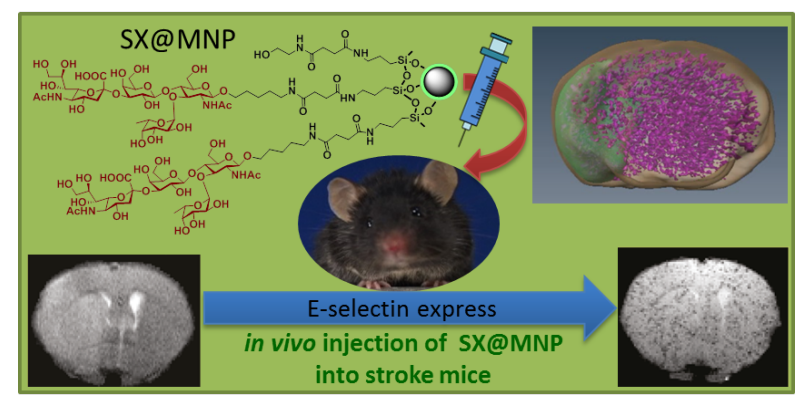

